\title{
Conservative management of bilateral femoral neck fractures in a child with autosomal dominant osteopetrosis
}

\author{
Andreas H. Krieg • B. M. Speth · H. Y. Won • \\ P. D. Brook
}

Received: 15 March 2007 / Published online: 17 July 2007

(C) Springer-Verlag 2007

\begin{abstract}
Management of minimally displaced femoral neck fractures in paediatric patients with autosomal dominant osteopetrosis (ADO) remains unclear as only small numbers have been reported. There are no detailed reports on successful conservative treatment. Common causes of failure in this particular area include non-union and development of coxa vara. Although there are no quantitative studies, case reports have influenced most authors to recommend operative treatment. It is well recognised that operative treatment of osteopetrotic bone is challenging. Problems arise intraoperatively due to the bone hardness, and postoperatively due to altered biomechanics and defective remodelling. This case of a child with ADO who suffered two asynchronous compression-side stress fractures in the femoral neck demonstrates that non-operative management can be satisfactory. After 8 weeks with partial weight-bearing the fractures were stable. At the latest follow-up 2.5 and 4 years after the fractures the patient presented with an excellent clinical and radiological outcome. There was no development of coxa vara.
\end{abstract}

Keywords Osteopetrosis $\cdot$ Fracture $\cdot$ Femoral neck . Children

\footnotetext{
A. H. Krieg $(\bowtie) \cdot$ B. M. Speth

Pediatric Orthopaedic Department,

University Children's Hospital Basel (UKBB),

Römergasse 8, 4005 Basel, Switzerland

e-mail: andreas.krieg@ukbb.ch

H. Y. Won · P. D. Brook

Department of Orthopaedic Surgery,

Women's and Children's Hospital,

72 King William Road, North Adelaide,

SA 5006, Australia
}

\begin{abstract}
Abbreviations
ADO Autosomal dominant osteopetrosis

NSA Neck-shaft angle
\end{abstract}

\section{Introduction}

Osteopetrosis is a rare inherited skeletal disease with defective osteoclast function. Osteoclast differentiation and activation is multifactorial and depends on progenitor cell competence as well as signals from osteoblasts and the extracellular microenvironment. Because of these complex interactions, bone resorption and remodelling can be inhibited in varying degrees of severity $[15,16,20]$. At least nine forms of osteopetrosis exist. Four clinical types of osteopetrosis have been reported in the literature: the malignant osteopetrosis (autosomal recessive) which appears during infancy and is often fatal from haematological manifestations, the intermediate form (autosomal recessive), the mild form (autosomal dominant) which is the biggest group, and a very rare fourth type associated with renal tubular acidosis [16].

Patients with the common mild variant of autosomal dominant osteopetrosis (ADO) have full life expectancy but suffer many orthopaedic problems. The main acute problem is the high incidence of fractures because of the highly brittle nature of such dense sclerotic bones. The fractures are commonly located at the neck of femur, the upper third of the femur shaft and tibia $[11,18,20]$.

Further problems are the development of coxa vara after femoral neck fractures and the high risk of osteomyelitis particularly at the mandible and after operative treatment $[11,13]$. The risk of osteomyelitis is higher due to immunodeficiency and less vascularity in the long bones [20].

While upper extremity fractures heal without complications with conservative treatment, surgical management is 
especially indicated for acute femoral neck fractures according to the Pediatric Orthopedic Society of North America (POSNA) survey [2]. The management of stress fractures of the femoral neck remains unclear.

Stress fractures are partial or complete disruptions of the bone secondary to an inability to withstand repetitive, nonviolent loads. There are two types of stress fractures: the "fatigue" fracture occurs when bone is in normal condition and "insufficiency" fracture be a result in bones with deficient mineral or elastic resistance [22]. Busch [5] recommended that femoral neck stress fractures in young active patients should be treated aggressively and may require internal stabilization.

To our knowledge, there are no detailed reports about successful conservative treatment of stress fractures in patients with osteopetrosis. Reported causes of failure include non-union and development of coxa vara in the femoral neck. Although there have been no quantitative studies, case reports have influenced most authors to recommend operative treatment in femoral neck fractures $[2,10,21]$. We present a patient managed non-operatively with bilateral asynchronous femoral neck fractures.

\section{Case report}

The patient presented at age five with a 3 days history of left leg pain and limping. There was no history of infection or trauma. Radiological examination of the left hip showed an osteosclerotic bone with cortical thickening and medullary calcifications. There was no evidence of a fracture. The family history revealed marble bones in both the maternal grandmother and a maternal uncle. Skeletal and genetic examination confirmed the diagnosis of ADO tarda.

For the assessment of a coxa vara we measured the neckshaft angle (NSA). At the time of first presentation at the age of five the patient had a bilateral NSA of $138^{\circ}$ (Table 1). This served as our baseline for subsequent follow-ups.

At the age of 6 years 7 months the patient presented with a history of few months pain in the right groin. The radiographs showed a femoral neck stress fracture at the compression-side (inferior aspect of the neck) with a medially displaced epiphysis (Fig. 1a). There was no associated causative trauma. At this age the NSA measured $136^{\circ}$ on both sides.

Treatment options were discussed with the parents and according to the literature research at this time $[2,5,10]$, we recommended internal fixation with a valgus osteotomy. The parents were concerned about the surgical complications and therefore asked for the patient to be managed conservatively as an option prior to surgery.

Consequently we treated the patient with partial weight bearing on crutches. After 8 weeks the patient had no limp or pain, and was able to resume all normal activities with a full range of motion in the right hip. Interestingly, X-rays and computer tomographs at that time did not show any callus formation (Fig. 1b).

Table 1 Femoral neck-shaft angles (NSA) for the patient and reference values

\begin{tabular}{llll}
\hline Age (years) & NSA R hip & NSA L hip & NSA: mean variation \\
\hline 5 & 138 & 138 & $129-143(4-5$ years $)$ \\
6.6 & $136^{\mathrm{b}}$ & 136 & $127-141$ (6-7 years) \\
6.8 & 132 & 133 & \\
8.3 & 128 & $128^{\mathrm{c}}$ & \\
8.5 & 128 & 128 & \\
9 & 128 & 128 & $126-140(9-13$ years $)$ \\
10 & 128 & 128 & \\
11 & 128 & 128 & \\
\hline
\end{tabular}

a According to Paley [19]

b Time of first

c Second fracture
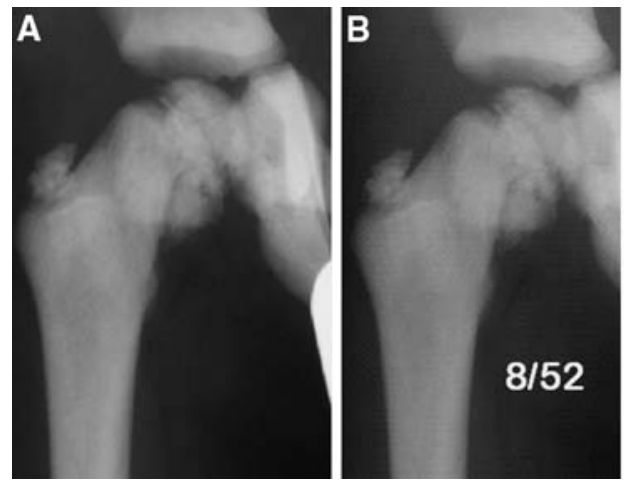

Fig. 1 a Femoral neck fracture with medial slip of the epiphysis at the time of first fracture. b Eight weeks later the X-Ray still shows the fracture line and no callus formation. $\mathbf{c}$ Four months after fracture with

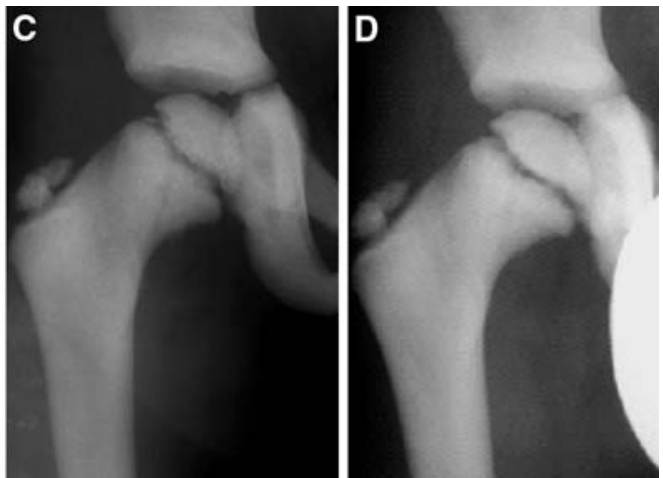

consolidation. d Complete remodelling of the right femoral neck 7 months after fracture 
Fig. 2 a Stress fracture at the left femoral neck. b Left hip 8 weeks after fracture and partial-weight bearing. c Patient at 5 months after fracture at the left-side with callus formation. d, e 1.5 years post $L$ fracture and 3 years post $\mathrm{R}$ fracture. $\mathbf{f}$ Complete remodelling of both femoral necks at the latest follow-up 2.5 years (left) and 4 years (right) after the fractures
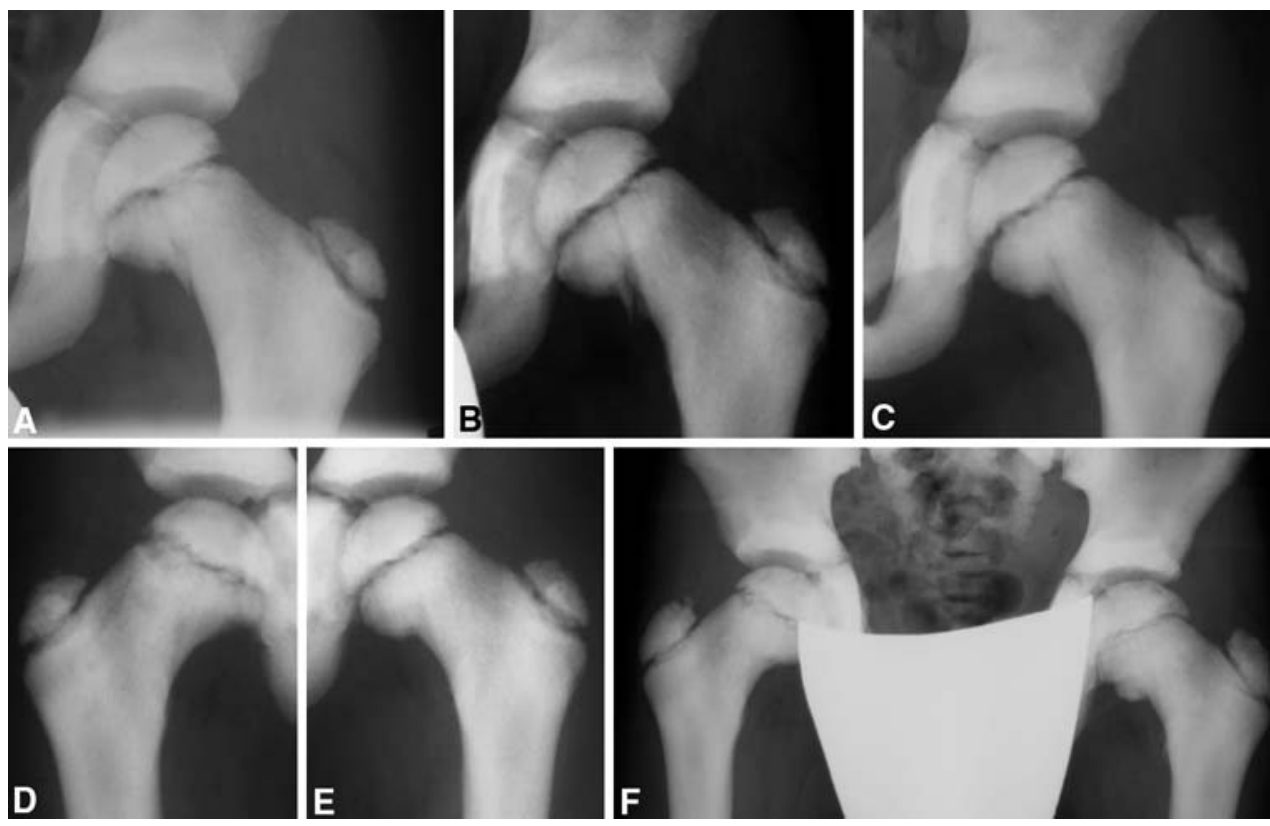

C

Radiographs after 4 months showed healing of the vertical fracture line (Fig. 1c), and at 7 months the fracture was united and the femoral neck remodelled (Fig. 1d).

Eighteen months after the right femoral neck stress fracture the patient started limping on the left side after playing at school without previous trauma. The radiographs of the 8 years 4 months old boy revealed a similar compressionside stress fracture (Salter-Harris type 2) at the left femoral neck (Fig. 2a). This fracture was also treated conservatively with partial weight bearing on crutches for a period of 8 weeks. At this time the radiographs did not show any callus formation at the left femoral neck (Fig. 2b) but he was free of pain with a full range of motion and weight bearing. First radiological signs of union at the left femoral neck were evident at the 5 months radiographs (Fig. 2c).

In another follow-up examination 9 months after the second femoral neck fracture the 9-year-old patient was asymptomatic with a full range of motion in both hips. Union was radiologically confirmed. After 2.5 years at the left side and after 4 years at the right side, the radiographs revealed completely remodelled femoral necks with a NSA of $128^{\circ}$ on both sides (Fig. $2 \mathrm{f}$ ).

Our analysis of the patient's NSAs and comparison with established age-matched ranges over a period of 4 years, did not show any evidence for the development of coxa vara (Table 1).

\section{Discussion}

Osteopetrosis, in its autosomal dominant form (ADO) is a benign variant and therefore often is incidentally discovered as in our case. Patients may be asymptomatic (45\%), present with osteomyelitis of the mandible (10\%), cranial nerve palsy (10-20\%), bone pain (20-25\%) or early hip osteoarthritis [2, 4, 6, 10,11, 17]. Our patient presented with hip pain in combination with limping. We defined the lesions as stress fractures as there was no associated causitive trauma.

Typical fracture patterns in osteopetrosis are transverse fractures perpendicular to the direction of stress because the dense but disorganised osteopetrotic bones cope with compression adequately, but are weak to tension.

Fracture healing has been studied histologically with specimens from up to 1 year post fracture [7]. In this report the biopsy showed that disorganised woven bone with no lamellar or Haversian systems persists with very low quantities of osteoclasts and osteocytes.

Surgical fixation is the recommended treatment especially in the femur neck. Internal fixation was thought to address the risks of non-union, subsequent coxa vara and avascular necrosis $[2,10,14,21]$. It is with these considerations that internal fixation was recommended as the treatment even for undisplaced fractures $[2,5]$. Series in the past using K-wires, Steinmann pins, blade plates, dynamic compression plates and screws have given reasonable results. Primary valgus osteotomy could also be performed if varus preceded the fracture $[2,11,19]$. Technical issues with drilling of the hard bone were commonly encountered [6, 1012, 18]. Longer operating time was needed and changing over of multiple drills helped to reduce the heat. Drill and chisel breakage were also reported [1].

Altered local biomechanics caused by the hardware created problems like peri-prosthetic fracture and re-fracture through screw holes after removal of the implant [12]. In Greene's report, the pin tracks were still evident on 
radiographs 2.5 years after their removal [10]. For osteopetrotic patients, the lack of remodelling in surrounding bone to cope with new stress distribution makes them more susceptible to this complication [7, 12].

Reported problems with conservative management includes delayed or non-union, with subsequent coxa vara deformity $[11,13]$. Bilateral proximal femoral non-union was reported by Alexander [1] in 1923.

Armstrong et al. [2] (POSNA survey) reported about closed reduction and internal fixation with pins or compression screws in four acute femoral neck fractures. Also they found no evidence of healing at 6 months in a series of three conservative treated femoral neck fractures in two patients. They were eventually internally fixed with primary valgus osteotomy. In their study two stress lesions were successfully treated with protection only.

Several authors have devised classifications for stress fractures of the femoral neck $[3,8,9]$. Stress fractures at the superior cortex of the femoral neck are tension fractures and are associated with a risk of displacement [8]. Fractures at the compression side at the inferior aspect of the femoral neck rarely show displacement. In bones with previously normal bone biology compression fractures are therefore usually treated conservatively by bed rest and a nonweight-bearing regimen.

In our case the patient was fully weight bearing after 8 weeks on both occasions because he was clinically asymptomatic by that time but still showed the fracture line without callus formation (Figs. 1b, 2b). The right side was consolidated after 4 months and the left side after 5 months (Figs. 1c, 2c). The early mobilization did not influence the healing nor cause development of coxa vara. Radiological analysis of radiographs consistently shows the NSA measurements to be within the normal range for age matched population (Table 1).

This case demonstrates that successful conservative treatment of undisplaced femoral neck stress fractures in ADO is possible. Management of femoral neck stress fractures in paediatric patients with ADO remains contentious. Both surgical and non-operative treatments present their own challenges. While coxa vara can develop, early prophylactic fixation or primary valgus osteotomy are probably unnecessary in children.

The outcome of our patient is clinically and radiologically successful. The lesson we involuntarily learned from this case led us to the conclusion that in the future we would treat a patient with the same problem in a stepwise regimen beginning with conservative treatment with regular radiographic follow-ups at 2 weeks, 1, 2, 4 and 6 months. In case of ongoing pain, absent callus formation after 6 months or progressive deformity we would consider an operative intervention. More reporting of experiences are needed in the future to help bring consensus.

\section{References}

1. Alexander WG (1923) Report of a case of so-called "marble bones" with a review of the literature and a translation of an article. Am J of Roentgenol 10:280-301

2. Armstrong DG, Newfield JT, Gillespie R (1999) Orthopedic management of osteopetrosis: results of a survey and review of the literature. J Pediatr Orthop 19(1):122-132

3. Blickenstaff LD, Morris JM (1966) Fatigue fracture of the femoral neck. J Bone Joint Surg Am 48(6):1031-1047

4. Bollerslev J, Mosekilde L (1993) Autosomal dominant osteopetrosis. Clin Orthop Relat Res 294:45-51

5. Busch MT (2001) Sports medicine in children and adolescents. In: Lovell WW, Winter RB, Morrissy RT, Weinstein SL (eds) Lippincott. Williams \& Wilkins, Philadelphia pp 1277-1279

6. Cameron HU, Dewar FP (1977) Degenerative osteoarthritis associated with osteopetrosis. Clin Orthop Relat Res 127:148-149

7. de Palma L, Tulli A, Maccauro G, Sabetta SP, del Torto M (1994) Fracture callus in osteopetrosis. Clin Orthop Relat Res 308:85-89

8. Devas MB (1965) Stress fractures of the femoral neck. J Bone Joint Surg Br 47(4):728-738

9. Fullerton LR Jr, Snowdy HA (1988) Femoral neck stress fractures. Am J Sports Med 16(4):365-377

10. Greene WB, Torre BA (1985) Femoral neck fracture in a child with autosomal dominant osteopetrosis. J Pediatr Orthop 5(4):483-485

11. Griss $P$, Schafer $T$ (1988) Therapy of bone and joint changes in Albers-Schonberg osteopetrosis. Orthopade 17(5):411-419

12. Gupta R, Gupta N (2001) Femoral fractures in osteopetrosis: case reports. J Trauma 51(5):997-999

13. King RE, Lovejoy JF Jr (1973) Familial osteopetrosis with coxa vara. A case report J Bone Joint Surg Am 55(2):381-385

14. Lam SF (1971) Fractures of the neck of the femur in children. J Bone Joint Surg Am 53(6):1165-1179

15. Marks SC Jr., Schmidt CJ (1978) Bone remodeling as an expression of altered phenotype: studies of fracture healing in untreated and cured osteopetrotic rats. Clin Orthop Relat Res 137:259-264

16. McCarthy EF, Frassica FJ (2000) Genetic diseases of bones and joints In: Pathology of bone and joint disorders. WB Saunders, Philadelphia

17. McKusick VA (1972) Heritable disorders of connective tissue. Mosby, St. Louis, Mo

18. Milgram JW, Jasty M (1982) Osteopetrosis. A morphological study of twenty-one cases. J Bone Joint Surg Am 64(6):912-929

19. Paley D (2002) Principles of deformity correction. Springer, Berlin

20. Shapiro F (1993) Osteopetrosis. Current clinical considerations. Clin Orthop Relat Res 294:34-44

21. Song KS, Kim HK (2005) Femoral neck fracture in a child with autosomal-dominant osteopetrosis: failure of spica cast treatment and successful outcome by internal fixation. J Orthop Trauma 19(7):494-497

22. St Pierre P, Staheli LT, Smith JB, Green NE (1995) Femoral neck stress fractures in children and adolescents. J Pediatr Orthop 15(4):470-473 